\title{
REVISIÓN SISTEMÁTICA DE LITERATURA \\ EN EL USO DE FLUIDOS SUPERCRÍTICOS \\ PARA LOGRAR UNA PRODUCCIÓN \\ MÁS LIMPIA EN LA INDUSTRIA TEXTIL
}

\author{
ChRISTINA ASCENCIO-RAMIREZ \\ https://orcid.org/0000-0001-6031-1634 \\ Agustina Micaela BuRgos \\ https://orcid.org/0000-0001-8716-2042 \\ KIMBERLY BRIGITTE LOPEZ-CRISOSTOMO* \\ https://orcid.org/0000-0001-7939-5305 \\ Universidad de Lima, Perú
}

Recibido: 6 de agosto del 2020 / Aprobado: 13 de agosto del 2020

doi: https://doi.org/10.26439/ing.ind2020.n039.4918

RESUMEN: Mediante una revisión sistemática de literatura, se buscó determinar el potencial de los fluidos supercríticos en la industria textil para alcanzar una producción más limpia. Para ello, se realizó un análisis integral de los diversos artículos de investigación relevantes, tomando como base aquellos cuyos resultados arrojaron resultados positivos para el medio ambiente en cuanto al uso eficiente de recursos. Se obtuvo entonces que la tecnología que abarca el empleo de fluidos supercríticos es el sustituto perfecto de aquellos subprocesos textiles húmedos dentro de la tintorería que, por su naturaleza, produzcan efluentes contaminados y posean un alto consumo de energía.

Palabras clave: fluidos supercríticos / industria textil / producción más limpia / revisión sistemática de literatura

\footnotetext{
*Correo electrónico: christina.lizzet@gmail.com, agussburgoss@hotmail.com,kim_1899@hotmail.com
} 


\section{A SYSTEMATIC LITERATURE REVIEW ON THE USE OF SUPERCRITICAL FLUIDS FOR CLEANER PRODUCTION IN THE TEXTILE INDUSTRY}

ABSTRACT: A systematic literature review sought to determine the potential of supercritical fluids in the textile industry to achieve cleaner production. To this end, a comprehensive analysis of various important research articles was carried out, taking as a basis those whose results were positive for the environment in terms of efficient use of resources. It was found that the technology that includes the use of supercritical fluids is the perfect substitute for those wet textile subprocesses within the dyeing industry that, by their nature, produce contaminated effluents and have a high-energy consumption.

Keywords: supercritical fluids / textile industry / cleaner production / systematic literature review 


\section{INTRODUCCIÓN}

En los últimos años, se ha hecho más frecuente la cuestión de cómo lidiar con los niveles de contaminación ambiental, que cada año van en aumento. $Y$ es, pues, que cada vez que se menciona el tema y se buscan culpables, generalmente se piensa en la industria petrolera o de transporte; sin embargo, se ha dejado de lado un sector que genera altos niveles de aguas residuales y es considerado el segundo mayor contaminante del mundo. Estamos hablando de la industria textil. Justamente, la Organización de las Naciones Unidas (2019) menciona que "la industria de la moda es responsable del 20 \% del desperdicio total de agua a nivel global" (párr. 20).

Ante esto surge el empleo de fluidos supercríticos (FSC) como alternativa sostenible ante métodos convencionales, puesto que conlleva una serie de ventajas tales como el hecho de que hace uso de disolventes no tóxicos, como el agua o el dióxido de carbono, o de baja toxicidad, como la acetona y el etanol, en proporciones menores de las empleadas en las técnicas tradicionales.

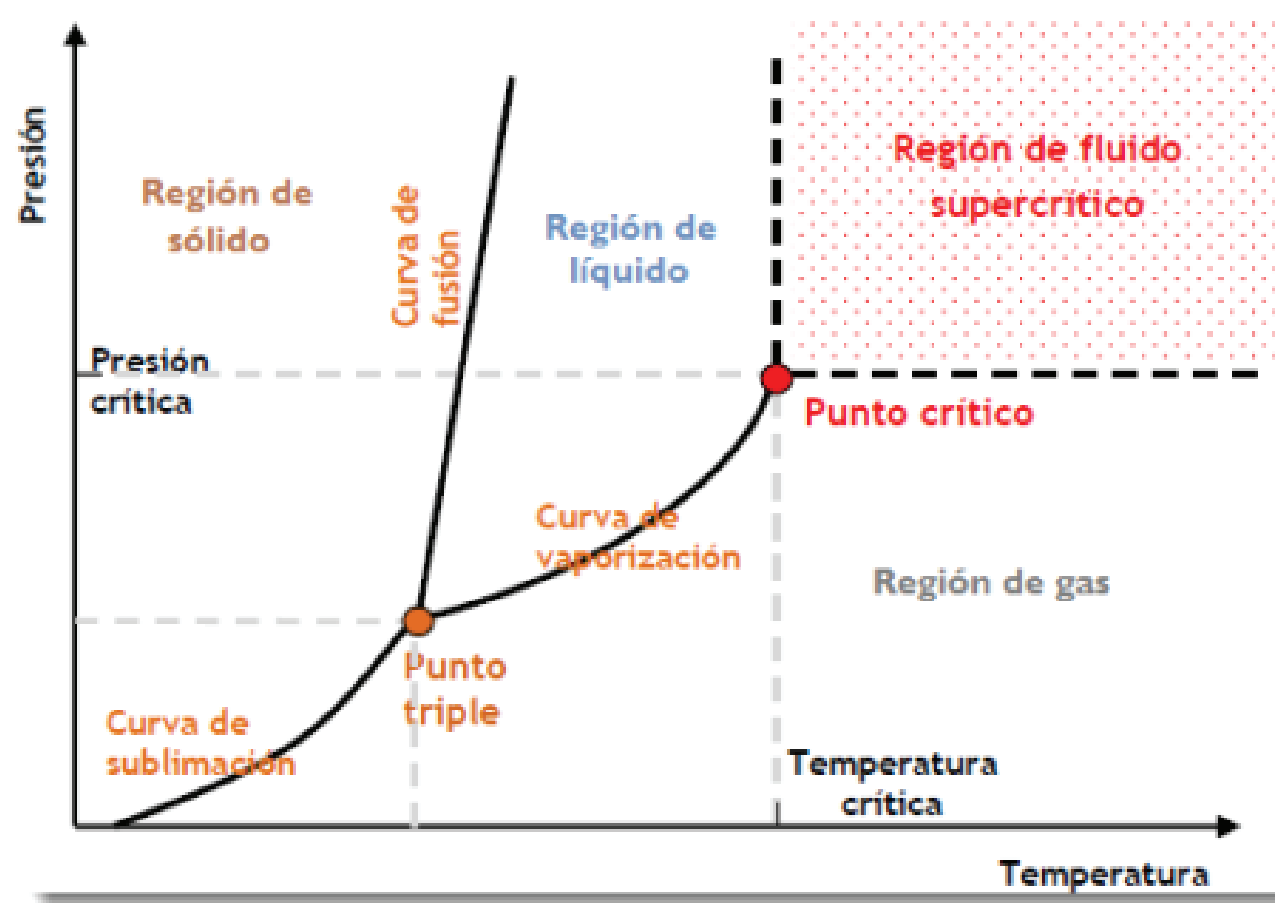

Figura 1. Región de fluido supercrítico

Fuente: Castaños (2015) 
De la figura se extrae que un FSC es una sustancia o mezcla de sustancias cuyas condiciones de presión y temperatura son superiores a su punto crítico. Los FSC muestran propiedades fisicoquímicas intermedias entre los líquidos y los gases que son responsables de su alta densidad y difusividad, así como baja viscosidad. Dichas propiedades otorgan a los FSC un elevado poder de penetración dentro de la matriz del soluto al actuar como disolventes (Guerrero, 2017, p. 8).

Ahondando en estas perspectivas, se han encontrado diferentes estudios con respecto a la utilización de FSC para procesos de teñido o blanqueo, entre otros, aplicando diversas metodologías, sustratos textiles, colorantes y muchas otras sustancias. Sin embargo, no existe hasta el momento una recopilación y análisis a profundidad de todos los métodos, variables consideradas y demás ítems dentro de cada investigación de manera transversal.

Cabe señalar en este punto el concepto de producción más limpia, como la aplicación continua de una estrategia ambiental preventiva integrada a los procesos, productos y servicios para aumentar la eficiencia global y reducir los riesgos para los seres humanos y el medio ambiente (Organización de las Naciones Unidas para el Desarrollo Industrial [ONUDI], s.f.).

De este modo, surge el siguiente planteamiento: ¿será posible alcanzar una producción más limpia en la industria textil global al 2020 aplicando la tecnología de los fluidos supercríticos en los procesos textiles? Para responder esto, en el presente trabajo se realizará una revisión sistemática de literatura en el uso de fluidos supercríticos en la industria textil, por lo cual se analizarán diversos artículos de investigación indexados en bases de datos.

\section{METODOLOGÍA}

Se empleará la base de datos Scopus, por su relevancia internacional, y el Research Engine Google Scholar, debido a la posibilidad de encontrar artículos de buena calidad y en idioma español.

Una vez definidas las bases de búsqueda, se pasó a definir los términos con los que se encontrará la cantidad de artículos iniciales. Se utilizaron los conceptos principales a los que hace referencia la pregunta de investigación. Asimismo, se identificaron distintas formas de escribir los términos, incluyendo abreviaturas y sinónimos. Finalmente, se verificó la inclusión de palabras claves, utilizadas previamente en artículos científicos relevantes.

De este modo, se realizó una búsqueda centrada fundamentalmente en la coincidencia de las palabras utilizadas con el título, palabras claves o resúmenes de los artículos ubicados en las diferentes bases de datos seleccionadas y se obtuvo lo siguiente: 


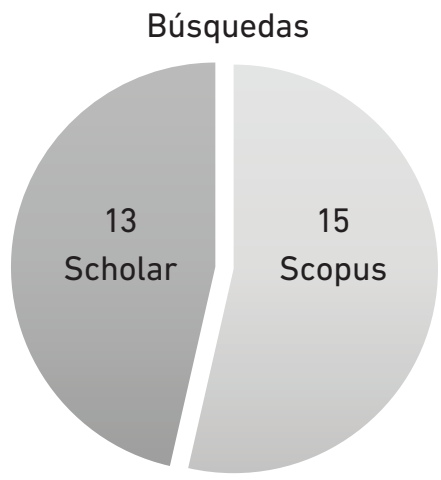

Figura 2. Resultados de Scopus y Scholar

\section{Elaboración propia}

\subsection{Método para la calificación de referencias a incluir en el estudio}

A los resultados se aplicaron los siguientes criterios de selección de artículos:

- Pertenecer a una revista académica indexada.

- Presentar resultados testeados con una metodología definida.

- Estar escrito en inglés o español.

- Pertenecer a los cuartiles Q1, Q2 o Q3.

- Haber sido redactado entre el 2013 y la actualidad.

- Tratar de la producción textil en general y la influencia de los FSC.

- Tener menos de treinta páginas.

- Tener estudios primarios.

- Estar referido a fluidos supercríticos en la industria textil.

Posteriormente, se hizo uso de la herramienta Mendeley; se leyeron en primer lugar los abstract de cada artículo para ver si cumplían o no con los criterios de búsqueda y selección presentados. Tras la revisión, los artículos que quedaron sumaban un total de trece, a los que posteriormente se aplicó una evaluación de calidad con el objeto principal de garantizar la integridad y fiabilidad de la información presentada en la revisión sistemática final. Para ello, se emplearon los criterios para la medición de la calidad de un artículo. Estos criterios tuvieron tres posibles respuestas: "Excelente" = 1, "Regular" $=0.5$, "Deficiente" $=0$. La evaluación de calidad se puede ver a continuación:

Solo se consideraron aquellos estudios con calidad aceptable, es decir, con una puntuación de calidad superior a 3,5 (50 \% de la puntuación perfecta) para la posterior extracción y síntesis de datos. Para el detalle de los papers, puede revisarse la siguiente tabla: 


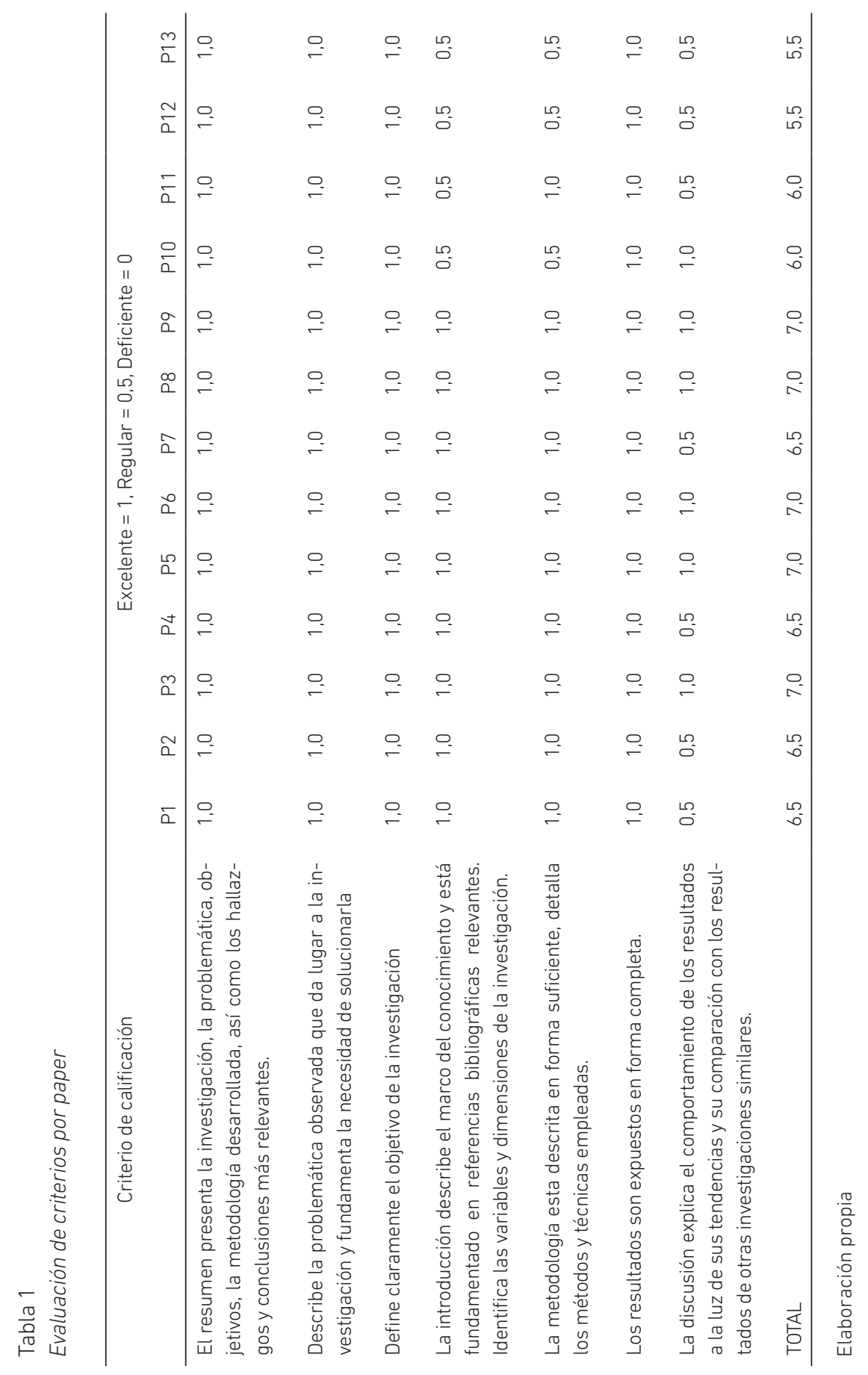


Tabla 2

Artículos seleccionados para el estudio

\begin{tabular}{|c|c|c|}
\hline n. ${ }^{\circ}$ paper & Título & Autor(es) \\
\hline P1 & $\begin{array}{l}\text { Single-step disperse dyeing and antimicrobial functiona- } \\
\text { lization of polyester fabric with chitosan and derivative in } \\
\text { supercritical carbon dioxide. }\end{array}$ & $\begin{array}{l}\text { Abate, Ferri, Guan, Chen, Ferreira } \\
\text { y Nierstrasz (2018) }\end{array}$ \\
\hline P2 & $\begin{array}{l}\text { Colouration and bio-activation of polyester fabric with } \\
\text { curcumin in supercritical } \mathrm{CO}_{2} \text { : Part I - Investigating co- } \\
\text { louration properties. }\end{array}$ & $\begin{array}{l}\text { Abate, Ferri, Guan, Chen y } \\
\text { Nierstrasz (2019) }\end{array}$ \\
\hline P3 & $\begin{array}{l}\text { Colouration and bio-activation of polyester fabric with } \\
\text { curcumin in supercritical } \mathrm{CO}_{2} \text { : Part II - Effect of dye con- } \\
\text { centration on the colour and functional properties. }\end{array}$ & $\begin{array}{l}\text { Abate, Zhou, Guan, Chen, Ferri y } \\
\text { Nierstrasz (2020) }\end{array}$ \\
\hline P4 & $\begin{array}{l}\text { Supercritical carbon dioxide as a green media in textile } \\
\text { dyeing: A review. }\end{array}$ & Abou Elmaaty y Abd El-Aziz (2017) \\
\hline P5 & $\begin{array}{l}\text { Supercritical Carbon-dioxide Assisted Dyeing of Textiles: } \\
\text { An Environmental Benign Waterless Dyeing Process. }\end{array}$ & Agrawal (2015) \\
\hline P6 & $\begin{array}{l}\text { A novel approach for textile cleaning based on supercri- } \\
\text { tical } \mathrm{CO}_{2} \text { and Pickering emulsions. }\end{array}$ & $\begin{array}{l}\text { Aslanidou, Tsioptsias y Panayiotou } \\
\text { (2013) }\end{array}$ \\
\hline P7 & $\begin{array}{l}\text { Waterless bleaching of knitted cotton fabric using super- } \\
\text { critical carbon dioxide fluid technology. }\end{array}$ & Eren, Avinc, Saka y Eren (2018) \\
\hline P8 & $\begin{array}{l}\text { Synthesis of a Novel Disperse Reactive Dye Involving a } \\
\text { Versatile Bridge Group for the Sustainable Coloration of } \\
\text { Natural Fibers in Supercritical Carbon Dioxide. }\end{array}$ & Fan, Zhang, Yan y Long (2019) \\
\hline P9 & $\begin{array}{l}\text { Synthesis of reactive disperse dyes containing halogena- } \\
\text { ted acetamide group for dyeing cotton fabric in supercri- } \\
\text { tical carbon dioxide. }\end{array}$ & Gao, Cui, Huang, Yang y Lin (2014) \\
\hline P10 & $\begin{array}{l}\text { Novel sustainable synthesis of dyes for clean dyeing of } \\
\text { wool and cotton fibres in supercritical carbon dioxide. }\end{array}$ & $\begin{array}{l}\text { Luo, White, Thompson, Rayner, } \\
\text { Kulik, Kazlauciunas, He y Lin (2018) }\end{array}$ \\
\hline P11 & $\begin{array}{l}\text { Development of a special SCFX-AnB3L dye and its appli- } \\
\text { cation in ecological dyeing of silk with supercritical car- } \\
\text { bon dioxide. }\end{array}$ & Yan, Zhang, Xiao, Shi y Long (2020) \\
\hline P12 & $\begin{array}{l}\text { Optimization of Eco-Friendly Reactive Dyeing of Cellulose } \\
\text { Fabrics Using Supercritical Carbon Dioxide Fluid with } \\
\text { Different Humidity. }\end{array}$ & Zhang, Zheng y Zheng (2018) \\
\hline P13 & $\begin{array}{l}\text { Supercritical } \mathrm{CO}_{2} \text { for color graphic dyeing: Theoretical in- } \\
\text { sight and experimental verification. }\end{array}$ & Zheng, Zhang, Du, Zhao y Ye (2015) \\
\hline
\end{tabular}

Elaboración propia 


\section{RESULTADOS}

\subsection{Descripción y análisis de las referencias en el estudio}

Se procedió a distribuir los papers en función de un número de páginas equitativo por miembro de trabajo, para luego clasificarlos, como se verá a continuación.

\subsection{Clasificación de las referencias en el estudio}

Se procedió a la descripción de las similitudes entre referencias, así como las diferencias que pudieran presentar, sistematizando y describiendo los aspectos que contribuyan a responder la pregunta de investigación propuesta en el estudio de investigación.

\section{Propósito}

El propósito de los papers evaluados se muestra en la tabla 3.

Tabla 3

Propósito de los artículos seleccionados para el estudio

\begin{tabular}{|c|c|}
\hline n. ${ }^{\circ}$ paper & Propósito \\
\hline P1 & $\begin{array}{l}\text { Reducir el costo de producción y la contaminación ambiental en los procesos de funcionaliza- } \\
\text { ción antimicrobiana con el uso de quitosano y derivados aplicando la ruta de teñido en dióxido } \\
\text { de carbono supercrítico }\left(\mathrm{scCO}_{2}\right)^{\text {. }}\end{array}$ \\
\hline P2-P3 & $\begin{array}{l}\text { Verificar el potencial del teñido en } \mathrm{scCO}_{2} \text { para la coloración de tejido de poliéster con curcu- } \\
\text { mina sin mordiente químico y pretratamiento del tejido, logrando una fabricación sostenible. }\end{array}$ \\
\hline P4 & $\begin{array}{l}\text { Destacar el papel de la tecnología de fluidos de } \mathrm{scCO}_{2} \text { en los procesos de teñido textil en tejidos } \\
\text { sintéticos y naturales, investigando el desarrollo de su alta eficiencia y teñido compacto para } \\
\text { ahorrar energía y agua en los procesos de fabricación. }\end{array}$ \\
\hline P5 & $\begin{array}{l}\text { Enfatizar la importancia del uso de } \mathrm{scCO}_{2} \text { en lugar de agua para teñido de textiles, especial- } \\
\text { mente los de fibras sintéticas, ya que éste no produce ningún drenaje y puede considerarse } \\
\text { como un enfoque de teñido respetuoso con el medio ambiente. }\end{array}$ \\
\hline P6 & $\begin{array}{l}\text { Se propone un novedoso método ecológico para la limpieza de textiles, basado en el } \mathrm{scCO}_{2} \\
\text { como disolvente y una suspensión acuosa como cosolvente. }\end{array}$ \\
\hline P7 & $\begin{array}{l}\text { Contribuir a alcanzar el hito de la minimización o abolición de los productos químicos y el agua } \\
\text { utilizados en procesos textiles acuosos comparando los métodos de blanqueo tradicionales } \\
\text { con aquellos en medio supercrítico. }\end{array}$ \\
\hline P8 & Mejorar las propiedades de coloración del colorante en el scCO \\
\hline P9 & $\begin{array}{l}\text { Garantizar una buena coloración en tejidos de algodón en medios supercríticos estudiando al } \\
\text { grupo funcional acetamina halogenada, capaz de reaccionar con las fibras de algodón y formar } \\
\text { enlaces químicos sin tratamiento previo ni cosolventes. }\end{array}$ \\
\hline P10 & $\begin{array}{l}\text { Encontrar una ruta para sintetizar los tintes dispersos azoicos y aplicarlos a un teñido de teji- } \\
\text { dos naturales utilizando tecnología supercrítica. }\end{array}$ \\
\hline P11 & $\begin{array}{l}\text { Sintetizar un colorante de una solidez especial usando fluido supercrítico para el teñido de } \\
\text { textiles, particularmente en el sustrato de seda. }\end{array}$ \\
\hline
\end{tabular}


(continuación)

P12 Demostrar que es posible mejorar el teñido en telas de celulosa con fluidos supercríticos haciendo énfasis en el parámetro humedad.

P13 Demostrar que el teñido gráfico con FSC es posible.

\section{Elaboración propia}

Los autores de estos artículos buscan entonces demostrar el potencial de los FSC en específicos procesos textiles usando diferentes aditivos, ya que cada uno de ellos otorgará distintas propiedades a las telas.

\section{Metodología empleada}

Para cumplir el propósito de su investigación, las metodologías encontradas en los diferentes estudios fueron:

Tabla 4

Metodología de los artículos seleccionados para el estudio

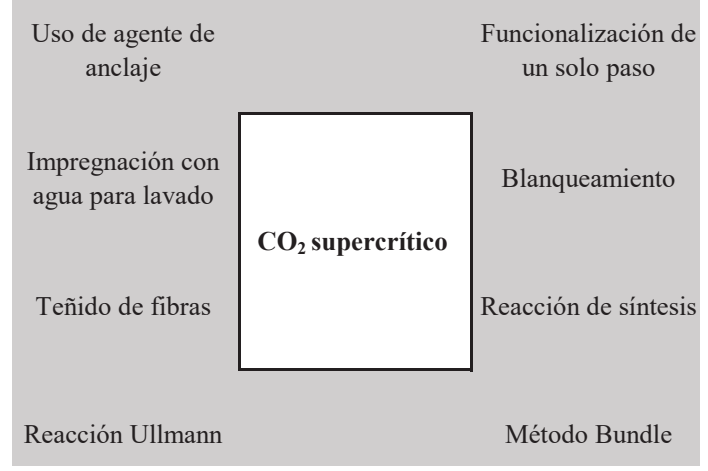

Elaboración propia

A continuación, se presenta una breve explicación de cada método:

- Funcionalización de un solo paso. Realizar el acabado de la tela en un solo proceso; por ejemplo, si se desea manufacturar una tela de color azul y antimicrobiana, usando un solo equipo se logrará este resultado, pues en él se incorporarán la tela, el pigmento y el agente antimicrobiano a la vez.

- Impregnación. Cuando la tela se sumerge a una solución para posteriormente ser escurrida usando de cilindros de presión, método que también se conoce como foulardado (Solé, 2016). 
- Uso de agente de anclaje. Consiste en usar un aditivo que aumente la fijación de otro aditivo en el material textil con el fin de mejorar sus atributos; por ejemplo, un incremento de la humectabilidad en la tela.

- Reacción de síntesis. Formación de un nuevo compuesto a partir de dos o más sustancias químicas; este método generalmente se usa en la industria textil para encontrar colorantes que puedan adherirse mejor a las fibras naturales.

- Método Bundle. Sistema de teñido donde la tela se dobla en forma de abanico y se empaqueta con otras telas, otorgando un diseño con estilo a la prenda (Zheng et al., 2015).

- Reacción Ullmann. Reacción de acoplamiento mediante la cual se unen enlaces carbono-carbono a estructuras aromáticas utilizando polvo de cobre como catalizador (Fan et al., 2019).

- Blanqueamiento y teñido de fibras. Tipos de procesos textiles que se explicarán posteriormente.

Variables medidas

Tabla 5

Variables medidas en los artículos seleccionados para el estudio

\begin{tabular}{|c|c|c|c|c|}
\hline & Intensidad & Resistencia & $\begin{array}{l}\text { Propiedades } \\
\text { antimicrobianas }\end{array}$ & Presión \\
\hline \multirow[t]{2}{*}{ COLOR } & Firmeza & Fuerza & Durabilidad & Temperatura \\
\hline & Difusión & Nivelación & Protección UV & Tiempo \\
\hline
\end{tabular}

Elaboración propia

Entre todos estos parámetros y variables, destacan los siguientes:

- Fuerza del color. Concentración para el rendimiento del color.

- Nivelación del color. Uniformidad con la que se dispersa el colorante en la tela.

- Firmeza del color. Solidez del color en el tejido. Indica cuán fuertemente se anclan las moléculas del colorante al sustrato textil.

- Difusión del color. Hace referencia a la migración del colorante, es decir, la máxima dispersión del colorante en el sustrato textil. Se obtiene un mejor valor si previamente se han realizado procesos para ayudar a que dicho sustrato absorba mejor el colorante y este se propague a más cantidad de tela. Se conoce también como rendimiento del color. 
- Intensidad de color. Depende de delta de L en una lectura espectrofotométrica y mide el grado de intensidad de un color. Es directamente proporcional al porcentaje de la tricromía del color (si se quiere más intensidad se añade más colorante).

Asimismo, a lo largo de los papers se han medido otras variables en función de lo que se esperaba conseguir. Ejemplo de ello fueron la aplicación del quitosano (como aditivo para funcionalizar) y la curcumina (para teñir y funcionalizar) para aumentar las propiedades del sustrato final en cuanto a propiedades antibacterianas y protección UV. De igual modo se esperaba que estas propiedades tuvieran durabilidad frente a varias lavadas posimpregnación.

Finalmente, se obtuvo que:

Tabla 6

Variables operativas

\begin{tabular}{|c|c|c|}
\hline & Variables operativas óptimas & Impacto en el medio ambiente \\
\hline P1 & $\begin{array}{l}\text { Presión: } 25 \text { MPa Temperatura: } \\
120^{\circ} \mathrm{C} \text { Tiempo: } 60 \text { minutos }\end{array}$ & $\begin{array}{l}\text { Se reduce la contaminación ambiental en el acabado anti- } \\
\text { microbiano de los textiles al usar un compuesto bioactivo } \\
\text { natural. }\end{array}$ \\
\hline P2 & $\begin{array}{l}\text { Presión: } 25 \text { MPa Temperatura: } \\
120{ }^{\circ} \mathrm{C} \text { Tiempo: } 60 \text { min }\end{array}$ & $\begin{array}{l}\text { Excelentes resultados sin el uso de productos químicos } \\
\text { auxiliares que resultan necesarios en el proceso de teñi- } \\
\text { do convencional de tintes naturales. }\end{array}$ \\
\hline P3 & $\begin{array}{l}\text { Presión: } 25 \text { MPa Temperatura: } \\
120^{\circ} \mathrm{C} \text { Tiempo: } 60 \text { min }\end{array}$ & $\begin{array}{l}\text { El uso de tinte natural de curcumina no necesita de pre- } \\
\text { tratamiento del sustrato ni de productos químicos en el } \\
\text { teñido textil. }\end{array}$ \\
\hline P4 & $\begin{array}{l}\text { Presión: } 20 \text { MPa Temperatura: } \\
\text { 119,85-129,85 C Tiempo: } 60 \text { min }\end{array}$ & $\begin{array}{l}\text { El scCO2 no es tóxico, los tintes se pueden reutilizar y no } \\
\text { se requiere ningún otro químico; con ello se ahorra ener- } \\
\text { gía y agua en los procesos de fabricación. }\end{array}$ \\
\hline P5 & $\begin{array}{l}\text { Presión: } 25 \text { MPa Temperatura: } \\
130{ }^{\circ} \mathrm{C} \text { Tiempo: } 60 \text { minutos }\end{array}$ & $\begin{array}{l}\text { Al teñir la fibra en } \mathrm{scCO}_{2} \text {, este se comprimió, reciclándose } \\
\text { y se utilizó nuevamente para la aplicación de colorante en } \\
\text { el sustrato de poliéster, evitando el desperdicio de agua. }\end{array}$ \\
\hline P6 & $\begin{array}{l}\text { Presión: } 20 \text { MPa Temperatura: } \\
40 \text { C Tiempo: } 60 \text { minutos }\end{array}$ & $\begin{array}{l}\text { Con el novedoso método para la limpieza de textiles } \\
\text { basados en el dióxido de carbono supercrítico como } \\
\text { disolvente y una suspensión acuosa como cosolvente se } \\
\text { minimizó el consumo de energía y de } \mathrm{CO}_{2} \text {. }\end{array}$ \\
\hline P7 & $\begin{array}{l}\text { Presión: } 18 \mathrm{MPa} \text {, Temperatura: } \\
80^{\circ} \mathrm{C} \text {, Tiempo: } 20 \mathrm{~min}\end{array}$ & $\begin{array}{l}\text { Durante el blanqueo de prendas se evita el uso de sosa } \\
\text { cáustica, estabilizadores de peróxido de hidrógeno y agua, } \\
\text { evitando efluentes contaminados. }\end{array}$ \\
\hline P8 & $\begin{array}{l}\text { Presión: } 20 \text { MPa, Temperatura: } \\
120^{\circ} \mathrm{C} \text {, Tiempo: } 90 \text { min }\end{array}$ & $\begin{array}{l}\text { Se mejoraron las propiedades de un colorante para teñir } \\
\text { en medio supercrítico, facilitando productos que favorez- } \\
\text { can el uso del teñido supercrítico. }\end{array}$ \\
\hline P9 & $\begin{array}{l}\text { Presión: } 20 \text { MPa, Temperatura: } \\
120^{\circ} \mathrm{C} \text {, Tiempo: } 180 \mathrm{~min}\end{array}$ & $\begin{array}{l}\text { Al sintetizar tintes reactivos se amplió el conocimiento } \\
\text { necesario para teñir con } \mathrm{scCO}_{2} \text { sin necesidad de trata- } \\
\text { mientos previos ni cosolventes. }\end{array}$ \\
\hline
\end{tabular}




\begin{tabular}{|c|c|c|}
\hline P10 & $\begin{array}{l}\text { Presión: } 14 \mathrm{MPa} \text {, Temperatura: } \\
90{ }^{\circ} \mathrm{C} \text {, Tiempo: } 60 \mathrm{~min}\end{array}$ & $\begin{array}{l}\text { Al sintetizar tintes reactivos dispersos, se obtuvo mayor } \\
\text { gama de colores de tintes que permiten alcanzar mayor } \\
\text { eficiencia en el teñido supercrítico. }\end{array}$ \\
\hline P11 & $\begin{array}{l}\text { Presión: } 20 \text { MPa, Temperatura: } \\
130{ }^{\circ} \mathrm{C} \text {, Tiempo: } 60 \text { min }\end{array}$ & $\begin{array}{l}\text { Se amplió el conocimiento para el teñido de seda median- } \\
\text { te un tinte especial para medios supercríticos, aumentan- } \\
\text { do la eficacia del sistema. }\end{array}$ \\
\hline P12 & $\begin{array}{l}\text { Presión: } 20 \text { MPa, Temperatura: } \\
90{ }^{\circ} \mathrm{C} \text {, Tiempo: } 60 \mathrm{~min}\end{array}$ & $\begin{array}{l}\text { Se modificó adicionalmente la humedad al } 5 \text { \% para au- } \\
\text { mentar la eficiencia del teñido supercrítico y minimizar } \\
\text { desperdicios. }\end{array}$ \\
\hline P13 & $\begin{array}{l}\text { Presión: } 24 \mathrm{MPa} \text {, Temperatura: } \\
120^{\circ} \mathrm{C} \text {, Tiempo: } 30 \mathrm{~min}\end{array}$ & $\begin{array}{l}\text { Se logró una combinación de dos tintes visibles en } \\
\text { tela sin el desperdicio de ninguno de ellos por medio } \\
\text { del teñido supercrítico, ampliando las posibilidades de } \\
\text { aplicabilidad. }\end{array}$ \\
\hline
\end{tabular}

Elaboración propia

\section{Tipo de fluido supercrítico empleado}

El FSC usado en todos los papers es el $\mathrm{CO}_{2}$ ya que es barato, no tóxico, no inflamable e inerte en condiciones críticas. Tiene la cualidad de hinchar y plastificar las telas con características hidrofóbicas, permitiendo el teñido de las fibras textiles (en especial las artificiales) y la dispersión del colorante sin la necesidad de usar agentes químicos; además, el $\mathrm{ScCO}_{2}$ es reutilizable junto a los tintes sobrantes y no requiere un proceso de secado posterior (Abate et al., 2019).

\section{Subproceso textil}

Aquellos subprocesos textiles estudiados a lo largo de los artículos que involucran el uso de fluidos supercríticos se muestran en la figura 3.

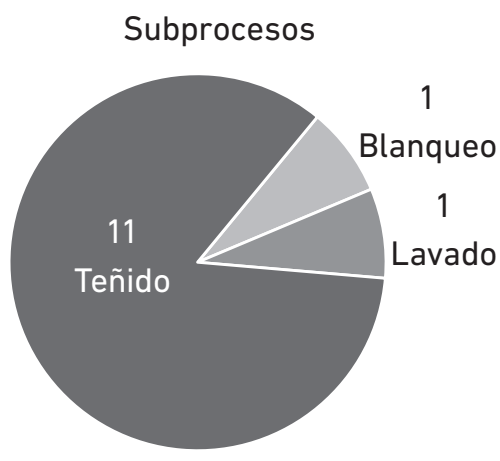

Figura 3. Subprocesos textiles de los artículos seleccionados para el estudio

Elaboración propia 
A continuación, se presenta una breve explicación de cada subproceso textil:

- Teñido supercrítico. Por medio de equipos de alta presión se impregna un sustrato textil (generalmente en forma de rollo), con colorantes de diversos tipos variando las condiciones de operación en función de estos últimos y del sustrato. Se hace en un solo paso y no requiere químicos auxiliares, mordientes ni el proceso de secado posterior al teñido convencional.

- Lavado supercrítico. Se basa en la emulsión pickering, que propone emplear partículas sólidas como agentes surfactantes para mezclar dos líquidos inmiscibles estabilizando al mismo tiempo dicha mezcla. Se logra, de este modo, limpiar diferentes tipos de suciedades formando emulsiones pickering con una mezcla acuosa de piedra caliza pulverizada y $\mathrm{scCO}_{2}$ y exponiendo los sustratos a limpiar a dicha emulsión estabilizada. Ejemplos de suciedades estudiadas en el paper 6 fueron: aceite de oliva, cera de abejas, pegamento de piel de conejo, pasta de remolacha y negro de carbón (Aslanidou et al., 2013).

- Blanqueo supercrítico. Es un paso previo al teñido aplicado en tejido de algodón (en el caso del paper 7) para la obtención de la coloración blanca deseada. Se realiza añadiendo peróxido de hidrógeno y ofrece resultados similares al blanqueo tradicional sin la necesidad de añadir estabilizadores de peróxido o soda cáustica (Eren et al., 2018).

\section{Sustratos textiles}

Los sustratos textiles se dividen en dos tipos: fibras sintéticas y fibras naturales. Las fibras sintéticas, también llamadas artificiales, provienen de compuestos derivados del petróleo. Los más utilizados en la fabricación textil son el tereftalato de polietileno (PET), la poliamida (PA), el poliacrilonitrilo (PAN) y el polipropileno (PP) (Abou y Abd, 2017). De todos ellos, el poliéster (PET), el nylon (PA) y el polipropileno (PP) fueron estudiados en los artículos seleccionados. Por otro lado, las fibras naturales son aquellas que, como su nombre lo dice, provienen de la naturaleza y pueden ser de origen vegetal o animal. Las más conocidas en la industria textil son el algodón, la lana, el bonote, la seda, el abacá, el cáñamo, etcétera. De todos ellos, el algodón, la lana y la seda fueron estudiados en los artículos seleccionados.

Es importante resaltar que las fibras sintéticas son las más usadas en los teñidos con $\mathrm{scCO}_{2}$ debido a sus características hidrofóbicas, su estructura compacta y alta cristalinidad (Abate et al., 2019); al contrario, las fibras naturales poseen características hidrofílicas, lo cual dificulta al fluido supercrítico romper los enlaces de hidrógeno presentes en la tela (Agrawal, 2015). 


\section{Sustratos}

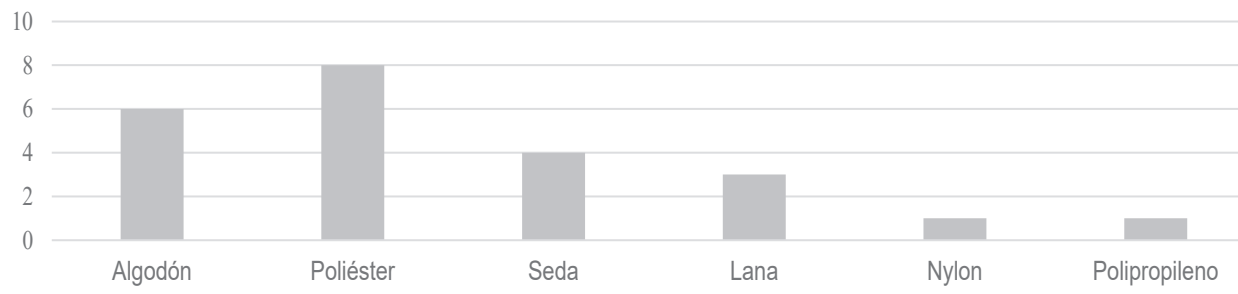

Figura 4. Sustratos textiles de los artículos seleccionados para el estudio

Elaboración propia

Nota. Como se aprecia, los sustratos empleados más comunes son el algodón y el poliéster.

Maquinaria y equipos empleados

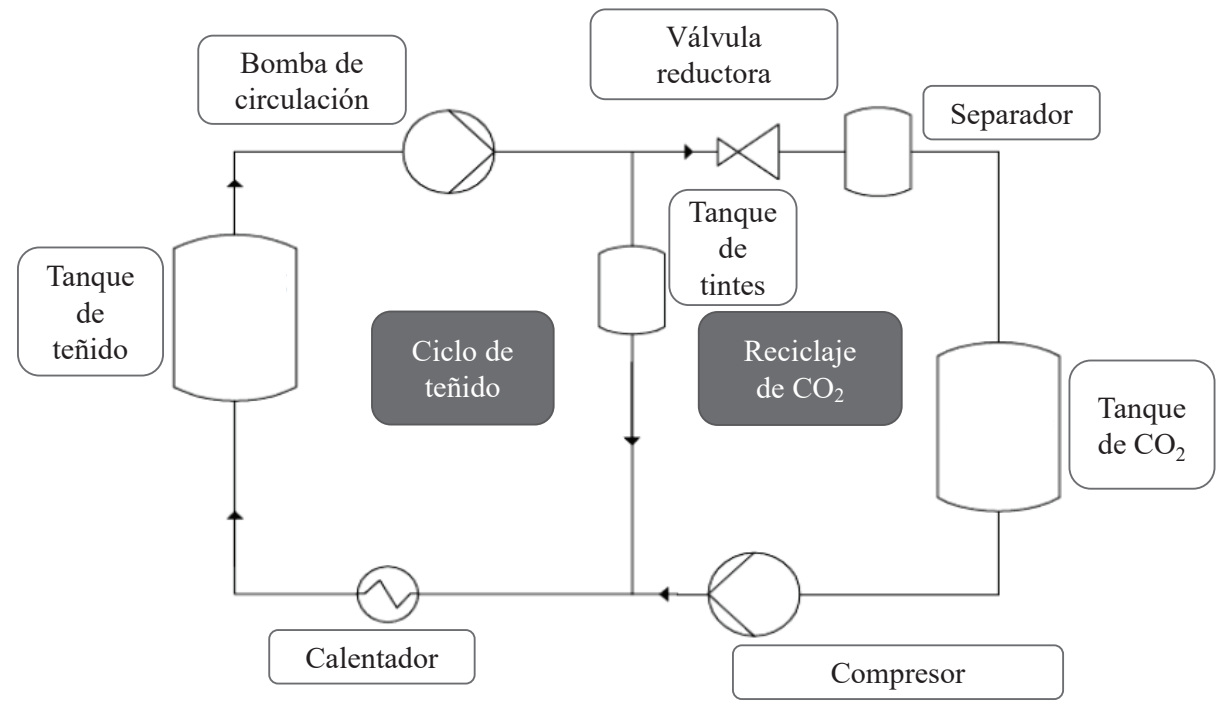

Figura 5. Diagrama de flujo simplificado para el sistema de teñido supercrítico de $\mathrm{CO}_{2}$

Elaboración propia

En el gráfico 1 se muestra un resumen del sistema de teñido de $\mathrm{scCO}_{2}$. El equipo de teñido contiene $\mathrm{CO}_{2}$ líquido precalentado, que recoge el tinte y realiza el teñido del sustrato textil. El tinte es absorbido por la fibra. Cuando se libera la presión, el $\mathrm{CO}_{2}$ se vuelve gaseoso y pierde su capacidad de disolverse, y los residuos de tinte se separan después de la licuefacción. $\mathrm{El} \mathrm{CO}_{2}$, libre de tinte, vuelve al tanque de recolección después de completar el proceso de teñido. Se detiene la circulación del $\mathrm{CO}_{2}$, la autoclave de teñido 
se despresuriza y el polvo de tinte no utilizado se deposita en el fondo de la máquina. Por lo tanto, el teñido supercrítico no produce ningún drenaje y puede ser considerado como un enfoque de teñido ecológico. Este sistema es aplicable a más subprocesos textiles.

\section{Tintes / aditivos empleados}

A lo largo de la evaluación de todos los papers, se presentan importantes tintes, blanqueadores o agentes funcionales empleados, como se muestra en la tabla 7.

Tabla 7

Tintes y aditivos empleados en los artículos seleccionados para el estudio

\begin{tabular}{lcll}
\hline \multicolumn{1}{c}{ Proceso textil } & n. & & Tinte \\
paper & & Aditivos \\
\hline Teñido de fibras sintéticas & P1 & Tintes dispersos & Quitosano y derivados \\
& P2-P3 & Curcumina & \\
P4 & Disperse Blue 79 & Poliglicol y acetona \\
Lavado de textiles & P5 & Colorante disperso & Ca(OH) \\
Blanqueamiento & P6 & & Peróxido de hidrógeno \\
Teñido de fibras naturales & P7 & 1-loroantraquinona, & \\
& P9 & Colorantes reactivo - dispersos & \\
Teñido de fibras naturales & P11 & Colorantes reactivo - dispersos & Sulfato de vinilo \\
& P10 & Colorante reactivo K-2RA & \\
\hline & P13 & Disperse Blue y Disperse Red & \\
\hline
\end{tabular}

Elaboración propia

Como se indica en la tabla 7, estos tintes y aditivos empleados son diferentes en los diversos papers evaluados; por ende, cada uno tiene su importancia y su manera de usarlo correctamente. Asimismo, los colorantes se pueden clasificar de la siguiente forma:

- Colorantes dispersos (poliéster). Son insolubles en agua y poseen una baja solubilidad en ella. Las partículas del colorante se disuelven en su forma monomolecular, y esta solubilidad está muy influenciada por las condiciones del medio, así como por otros factores propios, entre los cuales podemos nombrar el agua, el pH del medio, etcétera, además de las altas temperaturas de trabajo (Fajardo, 2016). 
- Colorantes reactivos (algodón). Son sustancias de estructuras no saturadas, orgánicas y solubles en agua. Se preparan comercialmente para tener uno o dos átomos de cloro que reaccionen con la celulosa formando enlaces covalentes con los grupos nucleófilos de la fibra. Estas sustancias son solubles en medio ácido, neutro o básico, poseen una estructura molecular no saturada (Peñafiel, 2011).

- Colorantes reactivos dispersos. Los tintes reactivos dispersos con una estructura química apropiada son clave para la coloración de las fibras naturales en el medio ecológico libre de agua del $\mathrm{scCO}_{2}$ (Fan y otros, 2019).

- Colorantes naturales. En los últimos años se ha reactivado el uso de colorantes naturales para el teñido de fibras textiles debido a la conciencia ambiental mundial y al énfasis que se ha dado a los procesos de producción más limpios y ecológicos. Una de las ventajas más importantes de los colorantes naturales es su compatibilidad con el medio ambiente; es decir, no causan ningún problema ambiental durante la etapa de producción y su uso mantiene el equilibrio ecológico. Muchos de los colorantes naturales tienen también propiedades multifuncionales y han sido considerados como agentes de acabado sostenibles y seguros en el desarrollo de textiles funcionales para una variedad de aplicaciones (Abate et al.,2019).

\section{Resultados}

Tabla 8

Principales resultados de los artículos seleccionados para el estudio

\begin{tabular}{|c|c|}
\hline $\begin{array}{c}\mathrm{n}^{\circ} \\
\text { paper }\end{array}$ & Resultados \\
\hline $\mathrm{P} 1$ & $\begin{array}{l}\text { Reducción de } 75-93 \% \text { de las bacterias (tasa de reducción bacteriana debe mejorarse para } \\
\text { aplicaciones médicas y de higiene). }\end{array}$ \\
\hline P2 & $\begin{array}{l}\text { A } 120^{\circ} \mathrm{C}, 25 \mathrm{MPa} \text { y una hora de tiempo de teñido, se logró una excelente intensidad, uniformidad } \\
\text { y solidez del color en la tela. }\end{array}$ \\
\hline P3 & $\begin{array}{l}\text { Los tejidos mostraron múltiples funcionalidades como las propiedades antibacterianas, antioxi- } \\
\text { dantes, solidez y de bloqueo de los rayos ultravioleta. }\end{array}$ \\
\hline P4 & $\begin{array}{l}\text { La aplicación de scCO2 en el teñido es un éxito para las fibras sintéticas, excepto para el propi- } \\
\text { leno, que necesita más investigación para encontrar tintes adecuados y condiciones de trabajo } \\
\text { más fáciles. }\end{array}$ \\
\hline P5 & $\begin{array}{l}\text { Tiempos de teñido más cortos; el poliéster ya no necesita tratamiento previo. Esta tecnología } \\
\text { se sigue considerando de última generación y no se encuentra fácilmente a escala comercial } \\
\text { en la actualidad. }\end{array}$ \\
\hline
\end{tabular}

(continúa) 
(continuación)

P6 El proceso de limpieza es más eficiente que otros para diferentes tipos de suciedad. El color de las muestras no se vio afectado. El proceso propuesto puede caracterizarse como un método de limpieza general.

P7 Comparado con el método de blanqueo tradicional sin la adición de estabilizante y sosa, el método con scCO2 ofreció valores similares de blancura y a $80{ }^{\circ} \mathrm{C}$ mejoró la blancura (30 \% y $51 \%$ ) para cada tipo de concentración de peróxido.

P8 Fijación de las moléculas de colorante absorbidas de 97,69 \% y de 89,76\% para la lana y la seda, respectivamente. El algodón obtuvo una menor eficiencia de fijación de 49,37 \%, reflejado en la baja intensidad de color. Se recomienda un proceso optimizado para sintetizar el nuevo tinte diseñado.

P9 La solidez al teñido y al roce cumplieron los requisitos, pero la solidez al lavado puede mejorar. Los tintes 1 y 2 mostraron los valores más altos de fijación. Los colores de la tela teñida con los tintes 2, 4 y 6 eran más profundos. Los tintes con el grupo bromoacetilo tuvieron mejor fijación en el algodón.

P10 A $90^{\circ}, 140$ bar y 60 minutos se produce un tinte uniforme con alta resistencia del color y propiedades de solidez, se obtiene un 99,4\% de fijación para la lana.

P11 Muy buenos comportamientos de adsorción, absorción, fijación y una alta solidez en la coloración. La mayor intensidad de color se alcanzó en la seda.

P12 La intensidad del color de las muestras teñidas mejoró con la variación de humedad del $\mathrm{scCO}_{2}$. Además, se obtuvo una aceptable resistencia al lavado, al frotamiento y a la luz; ergo, se redujo el tiempo de teñido.

P13 A $120^{\circ} \mathrm{C}$ y $24 \mathrm{MPa}$ se obtuvo el mejor coeficiente de difusión y el $\mathrm{scCO}_{2}$ con alta difusión y solubilidad favoreció la capilaridad de los tintes al interior de las fibras.

Elaboración propia

\section{DISCUSIÓN}

Pasando a explicar analizar los resultados para dar respuesta a nuestra pregunta de investigación, las referencias bibliográficas evaluadas afirman que el uso de los FSC en la industria textil traería beneficios ambientales y económicos al disminuir la generación de aguas residuales y compuestos químicos en la producción, lo que a la vez permite un ahorro significativo de energía (Abate et al., 2019). Por ello, se aprecia notoriamente que el propósito de los investigadores es comprobar el potencial de los fluidos supercríticos en los diferentes procesos textiles, pues ya que esta es una tecnología relativamente nueva, es necesario verificar que las propiedades otorgadas a la tela cumplan o sobrepasen los requisitos mínimos de calidad y, en caso no se logre, investigar metodologías para la mejora de estos parámetros con el fin de realizar una 
producción más limpia. Por ejemplo, en el paper 8 se menciona que la coloración de las fibras naturales (algodón, lana y seda) mediante dióxido de carbono supercrítico es compleja debido a que estos sustratos tienen una alta afinidad por el agua; por ello, los autores buscan mejorar las propiedades de coloración al emplear un tinte especial en el teñido textil (Fan et al., 2019).

Metodologías. Las más empleadas en los artículos fueron impregnación y reacción de síntesis. Esto se debe a que, para que el material textil obtenga el color deseado, la impregnación es una técnica básica para el teñido de las telas debido a su uso en producciones continuas y efectividad. Asimismo, diversos papers analizados se enfocaban en la creación de nuevos tintes para mejorar la eficiencia en el teñido de las fibras textiles y para lograr esto se debe realizar reacciones de síntesis.

Variables. Las más comunes son el tiempo, con un rango de veinte minutos a doce horas; la presión, con un rango de 180 a 250 bares, y la temperatura, con un rango de $40^{\circ} \mathrm{C}$ a $140{ }^{\circ} \mathrm{C}$. La mayoría de los estudios analizados converge en el hecho de que se trabaja a distinta temperatura y presión para un mismo tiempo para evaluar aquellas en las que obtiene un resultado óptimo en cuanto al contacto entre el substrato textil y los tintes, colorantes, blanqueadores o agentes funcionales empleados. Asimismo, cabe resaltar que, según el paper 2 , el comportamiento del teñido estaba mucho más influenciado por la presión del sistema seguido por la temperatura del proceso (Abate et al., 2019).

Subproceso textil. El más estudiado a lo largo de los artículos fue el teñido supercrítico, ya que demostró ser una coloración anhidra la cual incluye la utilización de menos energía y productos químicos, lo que supone un potencial de hasta un $50 \%$ menos de costos operativos (Eren et al., 2018, p. 2). Sin embargo, otros procesos están siendo estudiados como el blanqueo o el lavado, como procesos de pre y postratamiento de la tela antes de ser teñida por el ahorro que permite en agua dulce.

Sustratos textiles. El algodón resaltó como uno de los más comunes entre los artículos por tratarse de la fibra natural más producida y utilizada con un uso popular en el mundo entero, tal y como lo indica el paper 7 (Eren et al., 2018). Asimismo, resultó de interés testear en poliéster ya que, según el paper 2, el poliéster (PET) es la fibra textil más importante en términos de volumen y valor del producto en el mercado debido a sus importantes propiedades y a su versatilidad en diversas aplicaciones (Abate et al., 2019). También es el material textil más investigado en lo que respecta a la tintura de $\mathrm{scCO}_{2}$, ya que es una fibra hidrófoba de estructura compacta y alta cristalinidad que permite una coloración a altas temperatura y presión.

Fluido supercrítico empleado. Se optó en todos los papers por $\mathrm{CO}_{2}$, ya que según el paper 1 , en el estado supercrítico el $\mathrm{CO}_{2}$ tiene una baja viscosidad y difusividad similar a la de los gases, todo esto combinado con una alta densidad y un poder solvente similar 
al de los líquidos (Abate et al., 2018). Estas propiedades ayudan a adquirir un alto poder solvente que puede ser fácilmente ajustado variando la presión y la temperatura del sistema. Adicionalmente, según el paper 3 , el $\mathrm{CO}_{2}$ utilizado como solvente proviene de una emisión industrial que es esencialmente barata, abundante, no tóxica y no inflamable, y tiene una temperatura crítica fácilmente alcanzable $\left(31^{\circ} \mathrm{C}\right)$ y una presión crítica de 7,4 MPa (Abate et al., 2020).

Maquinaria. Aquella común a todos los papers fue un tanque de teñido con un cilindro hueco perforado dentro del cual se aplica presión y temperatura para obtener $\mathrm{scCO}_{2}$. Asimismo, se contó con un separador, el cual recupera la mayor cantidad del FSC luego del subproceso respectivo, y recircula el exceso de colorante y otros residuos. En la mayoría de papers los instrumentos más usados se destinan al control de temperatura, presión e intensidad de color, ya que de estos depende, en gran medida, la calidad y eficiencia del teñido. Una temperatura o presión incorrecta puede hacer que una tela, que se esperaba fuese de primera calidad, pase a ser de segunda o tercera calidad, debido a que se destiñe o no logró la intensidad de color requerido. Además, según el paper 1, después del teñido, el $\mathrm{CO}_{2}$ y los tintes sobrantes se recuperan y no se necesita ningún paso de secado, lo cual es económico (Abate et al., 2018).

Tintes y aditivos. Al emplearse sustratos como algodón y poliéster, los tintes más comunes fueron reactivos (para el algodón) y dispersos (para el poliéster). Los reactivos son colorantes que permiten mediante una reacción covalente adherirse a la fibra en temperaturas de entre 20 y $100^{\circ} \mathrm{C}$, mientras que los dispersos actúan comúnmente en un medio ácido $(4,5)$ y son poco solubles en agua a $130{ }^{\circ} \mathrm{C}$. Asimismo, se emplearon colorantes naturales que, según el paper 3 , poseen propiedades multifuncionales y han sido considerados como agentes de acabado sostenibles y seguros en el desarrollo de textiles funcionales para una variedad de aplicaciones (Abate et al., 2020). En este último caso, usando el método de impregnación con FSC, no fue necesario el uso de mordientes (metales pesados para fijar el colorante en métodos tradicionales). Como punto adicional, según el paper 4, en el teñido supercrítico se espera que la concentración de tinte requerida para un tono específico sea menor que en métodos de teñido húmedos (Abou y Abd, 2017).

Resultados. Se pudo observar que, al aplicar esta novedosa propuesta de teñido con $\mathrm{scCO}_{2}$, se obtienen resultados sumamente satisfactorios estando el incremento de la resistencia al lavado y el aumento del rendimiento de color entre los principales hallazgos. Además, se observó la obtención de un tinte uniforme con alta resistencia del color y excelentes propiedades de solidez (cumpliendo los requisitos para prendas de vestir), fijación y profundidad. Otro aspecto que vale la pena resaltar es una importante mejora en la reducción bacteriana, que incluso llegó a un 60 \%. Por otro lado, para objetivos de blanqueamiento, se pudo notar un incremento considerable de blancura a la temperatura de $80^{\circ} \mathrm{C}$. Con todo, lo que limitaría la globalización de esta tecnología 
(mencionado en el paper 5 ) es la alta inversión de este tipo de sistemas en comparación de los procesos tradicionales; aun así, los beneficios económicos posteriores son importantes, ya que el consumo de agua disminuiría excesivamente, así como la generación de aguas residuales (Abou y Abd, 2017).

Finalmente, se plantea para una futura investigación que se lleven a cabo propuestas con el uso de FSC en los subprocesos textiles de modificación de superficies, desencolado, fijado, esponja, neutralizado y suavizado, pues estos son procesos aún sin mucha exploración y que tienen un alto consumo de agua en la producción de prendas.

\section{CONCLUSIONES}

- Mediante una revisión sistemática de literatura, se logró verificar que el uso de FSC en los procesos textiles permite alcanzar una producción más limpia en la industria textil.

- Se determinó un panorama del estado para comprender la aplicabilidad de los FSC en la actualidad, detallando maquinarias y métodos validados por los artículos estudiados.

- Se mapearon las fuentes de conocimiento más fiables para dar con la información más confiable luego de aplicar los filtros a la información.

- Como ventaja principal, el uso de fluidos supercríticos en la industria textil permite el ahorro de agua y bajo consumo de energía por ahorrar el proceso de secado. Como desventaja principal, aún hay pocos datos con respecto a la solubilidad de los diferentes tipos de colorante en el $\mathrm{scCO}_{2}$.

- Se determinó que es posible alcanzar una producción más limpia en la industria textil global al 2020 aplicando la tecnología de los FSC en los procesos textiles, siendo un aspecto crucial las variables de temperatura, tiempo y presión, las cuales varían en gran medida y necesitar estar predeterminadas según el tipo de sustrato, método, tipo de colorante y subproceso textil a llevar a cabo.

- Se encontró que la razón por que la tecnología supercrítica no se comercializa a gran escala es por los costos altos en los que se debe incurrir en este sistema; sin embargo, los artículos referenciados (gran mayoría publicados recientemente) demuestran el potencial de los FSC y los beneficios económicos que podrán generar su empleo. Además, si los científicos concentran sus esfuerzos en investigar nuevos procesos textiles en los que se pueda dar uso a los FSC, no cabe duda de que se tratará de una tecnología eficiente verde que toda empresa industrial debería pensar en emplear para ser responsables con el medio ambiente. 


\section{REFERENCIAS}

Abate, M. T.; Ferri, A.; Guan, J.; Chen, G. y Nierstrasz, V. (2019). Colouration and bio-activation of polyester fabric with curcumin in supercritical $\mathrm{CO}_{2}$ : Part I Investigating colouration properties. The Journal of Supercritical Fluids, 152. https://doi.org/10.1016/j.supflu.2019.104548

Abate, M. T.; Ferri, A.; Guan, J.; Chen, G.; Ferreira, J. A. y Nierstrasz, V. (2018). Singlestep disperse dyeing and antimicrobial functionalization of polyester fabric with chitosan and derivative in supercritical carbon dioxide. The Journal of Supercritical Fluids, 147, 231-240. https://doi.org/10.1016/j.supflu.2018.11.002

Abate, M. T., Zhou, Y., Guan, J., Chen, G., Ferri, A. y Nierstrasz, V. (2020). Colouration and bio-activation of polyester fabric with curcumin in supercritical $\mathrm{CO}_{2}$ : Part II Effect of dye concentration on the colour and functional properties. The Journal of Supercritical, 157. https://doi.org/10.1016/j.supflu.2019.104703

Abou Elmaaty, T. y Abd El-Aziz, E. (2017). Supercritical carbon dioxide as a green media in textile dyeing: A review. Textile Research Journal, 88(10), 1184-1212. https:// doi-org.ezproxy.ulima.edu.pe/10.1177/0040517517697639

Agrawal, B. J. (2015). Supercritical Carbon-dioxide Assisted Dyeing of Textiles: An Environmental Benign Waterless Dyeing Process. In International Journal of Innovative Research and Creative Technology, 1(2), IJIRCT. Recuperado de http:// www.ijirct.org/papers/IJIRCT1201043.pdf

Aslanidou, D.; Tsioptsias, C. y Panayiotou, C. (2013). A novel approach for textile cleaning based on supercritical $\mathrm{CO}_{2}$ and Pickering emulsions. The Journal of Supercritical Fluids, 76, 83-93. https://doi.org/10.1016/j.supflu.2013.02.005

Castaños, E. (17 de agosto de 2015). Cromatografía de fluidos supercríticos. Recuperado de https://cienciaonthecrest.com/2015/08/17/cromatografia-de-fluidos-super criticos/

Eren, S.; Avinc, O.; Saka, Z. y Eren, H. A. (2018). Waterless bleaching of knitted cotton fabric using supercritical carbon dioxide fluid technology. Cellulose, 25(10), 6247-6267. Recuperado de https://link-springer-com.ezproxy.ulima.edu.pe/article/10.1007/ s10570-018-2004-z

Fajardo, 0. (16 de octubre de 2016). Productos de limpieza para lavandería industrial. Conocimiento textil. Recuperado de https://conocimiento-textil.blogspot. com/2016/10/tintoreria-textil-tenido-colorantes-dispersos-poliester.html

Fan, Y.; Zhang, Y. Q.; Yan, K. y Long, J. J. (2019). Synthesis of a Novel Disperse Reactive Dye Involving a Versatile Bridge Group for the Sustainable Coloration of Natural Fibers in Supercritical Carbon Dioxide. Advanced Science, 6(1), 1-14. https:// doi-org.ezproxy.ulima.edu.pe/10.1002/advs.201801368 
Gao, D.; Cui, H. S.; Huang, T. T.; Yang, D. F. y Lin, J. X. (2014). Synthesis of reactive disperse dyes containing halogenated acetamide group for dyeing cotton fabric in supercritical carbon dioxide. The Journal of Supercritical Fluids, 86, 108-114. https://doi.org/10.1016/j.supflu.2013.12.006

Guerrero Muñoz, N. (2017). Estudio de un proceso de extracción e impregnación de extractos naturales en telas de poliéster [tesis de licenciatura, Universidad de Cádiz, España]. Repositorio institucional de la Universidad de Cádiz. Recuperado de https://rodin.uca.es/xmlui/handle/10498/19740

Luo, X., White, J., Thompson, R., Rayner, C., Kulik, B., Kazlauciunas, A., He, W. y Lin, L. (2018). Novel sustainable synthesis of dyes for clean dyeing of wool and cotton fibres in supercritical carbon dioxide. Journal of Cleaner Production, 199, 1-10. https://doi-org.ezproxy.ulima.edu.pe/10.1016/j.jclepro.2018.07.158

Organización de las Naciones Unidas para el Desarrollo Industrial (s.f.). Manual de producción más limpia. Recuperado de https://docplayer.es/10773854-Onudimanual-de-produccion-mas-limpia.html

Organización de las Naciones Unidas. (12 de abril de 2019). El costo ambiental de estar a la moda. Recuperado de https://news.un.org/es/story/2019/04/1454161

Peñafiel, S. (9 de junio de 2011). Influencia del suavizado con bases de ácidos grasos en el cambio de matiz en tejidos algodón $100 \%$ tinturados con colorantes reactivos de baja reactividad [tesis de licenciatura, Universidad Técnica del Norte, Ecuador]. Repositorio institucional de la Universidad Técnica del Norte. Recuperado de http://repositorio.utn.edu.ec/handle/123456789/631

Solé, A. (abril de 2016). Procesos de tintura por agotamiento e impregnación. Asolengin. Recuperado de https://asolengin.files.wordpress.com/2016/04/procesos-detintura-por-agotamiento-e-impregnacic3b3n.pdf

Yan, K.; Zhang, Y. Q.; Xiao, H.; Shi, M. W. y Long, J. J. (2020). Development of a special SCFX-AnB3L dye and its application in ecological dyeing of silk with supercritical carbon dioxide. Journal of $\mathrm{CO}_{2}$ Utilization, 35, 67-78. https://doi-org.ezproxy.ulima. edu.pe/10.1016/j.jcou.2019.09.004

Zhang, J.; Zheng, H. y Zheng, L. (2018). Optimization of Eco-Friendly Reactive Dyeing of Cellulose Fabrics Using Supercritical Carbon Dioxide Fluid with Different Humidity. Journal of Natural Fibers, 15(1), 1-10. https://doi-org.ezproxy.ulima.edu. pe/10.1080/15440478.2017.1302385

Zheng, L. J.; Zhang, J.; Du, B.; Zhao, Y. P. y Ye, F. (2015). Supercritical $\mathrm{CO}_{2}$ for color graphic dyeing: Theoretical insight and experimental verification. Thermal Science, 19(4), 1287-1291. https://doi.org/10.2298/TSCI1504287Z 